Workshop on Econophysics of the

International Conference on Statistical Physics (SigmaPhi2011)

International Journal of Modern Physics: Conference Series

Vol. 16 (2012) 116-135

(C) World Scientific Publishing Company

DOI: $10.1142 /$ S2010194512007829

\title{
MUSICAL MARKOV CHAINS
}

\author{
DIMA VOLCHENKOV*,‡ and JEAN RENÉ DAWIN ${ }^{\dagger}$ \\ * Center of Excellence Cognitive Interaction Technology, \\ Universität Bielefeld, Postfach 1001 31, \\ 33501 Bielefeld, Germany \\ $\dagger$ Department of Physics, Universität Bielefeld, \\ Postfach 1001 31, 33501 Bielefeld, Germany \\ ¥volchenk@physik.uni-bielefeld.de
}

\begin{abstract}
A system for using dice to compose music randomly is known as the musical dice game. The discrete time MIDI models of 804 pieces of classical music written by 29 composers have been encoded into the transition matrices and studied by Markov chains. Contrary to human languages, entropy dominates over redundancy, in the musical dice games based on the compositions of classical music. The maximum complexity is achieved on the blocks consisting of just a few notes ( 8 notes, for the musical dice games generated over Bach's compositions). First passage times to notes can be used to resolve tonality and feature a composer.
\end{abstract}

Keywords: Markov chains; entropy and complexity of music; first passage times.

\section{Introduction}

The Internet based economy calls for robust recommendation engines for appreciating and predicting the musical taste of customers, since any improvement in the accuracy of predictions might have an immense economic value. From one hand, studies of Markov chains aggregating pitches in musical pieces might provide a neat way to efficient algorithms for identifying musical features important for a listener. From another hand, the analysis of weighted directed graphs correspondent to the time-irreversible random walks defined on a finite set of states (pitches) belonging to a cyclic group (under the assumption of octave equivalency, the cyclic group is $\mathbb{Z} / 12 \mathbb{Z}$, as the perceived fundamental frequency of a sound $f=440 \mathrm{~Hz} \times 2^{n / 12}$, $n \in \mathbb{Z})$ is a daunting task for the contemporary theory of networks being therefore of a special theoretical interest.

Interactions between humans via speech and music constitute the unifying theme of research in modern communication technologies. As with music, speech and written language also have the sets of rules (crucial for establishing efficient communication) that determine which particular combinations of sounds and letters may or may not be produced. However, while communications by the spoken and written forms of human languages have been paid much attention from the very onset 
of information theory ${ }^{1,2}$, not very much is known about the relevant information aspects of music ${ }^{3}$. Although we use the acoustic channel in both music and speech, the acoustical and structural features we implement to encode and perceive the signals in music and speech are dramatically different, as "speech is communication of world view as the intellection of reality while music is communication of world view as the feeling of reality" 4 .

A Markov chain model allows to appraise tonal music as a generalized communication process, in which a composer sends a message transmitted by a performer to a listener. The applications of Markov chains in music have a long history dating back to 1757 (see Sec. 2). In modern times, the first computer program that used Markov chains to compose a string quartet (Illiac Suite) was developed in $1957^{5}$. The further developments of computer musical data formats also called for a formalization of musical events either in terms of frequency, duration, and intensity ${ }^{6}$, or in terms of pitch, duration, amplitude, instrument ${ }^{7}$ - in the both cases, the musical events were naturally treated as the states of hierarchical Markov chains. Although much work using Markov chains for compositional purposes have been done in so far, including a real-time interactive control of the Markov chains ${ }^{8}$, less researches have been focused on the detailed analysis of musical compositions by means of Markov chains. The reason for such a discrepancy is quite simple: Markov chains encoding musical compositions might be not ergodic thus being difficult to analyze.

Although stochastic techniques have the certain advantage over other compositional approaches polishing computer-generated music that might sound rather artificial otherwise, there has been some criticism of using Markov chains to create music. $\mathrm{In}^{9,10}$, it has been pointed that the reliance on random note selection tends to obscure the practices of music compositions and thus can not be considered as consistent with a composition of high quality music. Perhaps, partially in response to such a criticism, in ${ }^{11,12}$, Markov chains have been used as tools for the jazz improvisation analysis. Indeed, a melodic pattern generated randomly in accordance to a given aggregated transition matrix would hardly resemble the original composition, as being just a particular random realization over an ensemble of statistically identical musical pieces which we call the Musical dice game (MDG) throughout the paper.

In our work, we report some results on the Markov chain analysis of MDG encoded by the transition matrices between pitches in the MIDI representations of the 804 musical compositions attributed to 29 composers: J.S. Bach (371), L.V. Beethoven (58), A.Berg (7), J. Brahms (8), D. Buxtehude (3), F. Chopin (26), C. Debussy (26), G. Fauré (5), C. Franck (7), G.F. Händel (45), F. Liszt (4), F. Mendelssohn Bartholdi (19), C. Monteverdi (13), W.A. Mozart (51), J. Pachelbel (2), S. Rachmaninoff (4), C. Saint-Saëns (2), E. Satie (3), A. Schönberg (2), F. Schubert (55), R. Schumann (30), A. Scriabin (7), D. Shostakovitch (12), J. Strauss (2), I. Stravinsky (5), P. Tchaikovsky (5), J. Titelouze (20), A. Vivaldi (4), R. Wagner (8). The MIDI representations of many musical pieces are freely available on the $\mathrm{Web}^{13}$. 
The paper is organized as follows. In Sec. 2, we introduce the MDG as Markov chains. In Sec. 3, we discuss the MIDI representations of music pieces and the different methods to encode them into a Markov chain transition matrix. The encoding problem is not trivial, as ambiguities would arise provided a piece has more than one voice. Then, in Sec. 4, we consider the MDG defined over musical compositions as a generalized communication process, in which some amount of information is generated. The statistical analysis of the note sequences generated by the MDG based on Bach's chorales shows that patches consisting of 8 notes are characterized by the maximum past-future mutual information (complexity). While the elements of the Markov chain transition matrix indicate the possibility to consequently find the two notes in the musical score, an infinite number of powers of the transition matrix must be considered to estimate the eventual distance between them with respect to the entire structure of the MDG. First passage times to notes in the MDG discussed in Sec. 5 resolve the hierarchical relations between pitches. The frequency analysis of note occurrences alone is not enough to reliably resolve the tonality of a musical composition generated by the MDG, since recurrence times to notes are typically longer than first passage times. The phylogenetic classification of composers, with respect to the tone scale preferences are discussed in Sec. 6. We conclude in the last section.

\section{Musical dice game as a Markov chain}

A system for using dice to compose music randomly, without having to know neither the techniques of composition, nor the rules of harmony, named Musikalisches Würfelspiel (Musical dice game) had become quite popular throughout Western Europe in the $18^{\text {th }}$ century ${ }^{14}$. Depending upon the results of dice throws, the certain pre-composed bars of music were patched together resulting in different, but similar, musical pieces. "The Ever Ready Composer of Polonaises and Minuets" was devised by Ph. Kirnberger, as early as in 1757. The famous chance music machine attributed to W.A. Mozart (K 516f) consisted of numerous two-bar fragments of music named after the different letters of the Latin alphabet and destined to be combined together either at random, or following an anagram of your beloved had been known since 1787 .

Contrary to the alphabets used in human languages, the sets of pitches underlying the different musical compositions can be very distinct and may not overlap (even under chromatic transposition). The cardinality $R$ of the set of pitches used to compose a piece is one of its important characteristics, as the melody obviously centers around a few given notes when this cardinality is small, but calling for a wide range, from low to high pitches, otherwise. The cardinality $R$ changes from piece to piece demonstrating a tendency of slow growth, with the length of arrangement $N$. In Fig. 1, we have sketched how the number of different pitches $R$ used to compose a piece depends upon its size $N$. The data collected over 610 pieces created by the six classical music composers show that the growth can be well approximated by a 


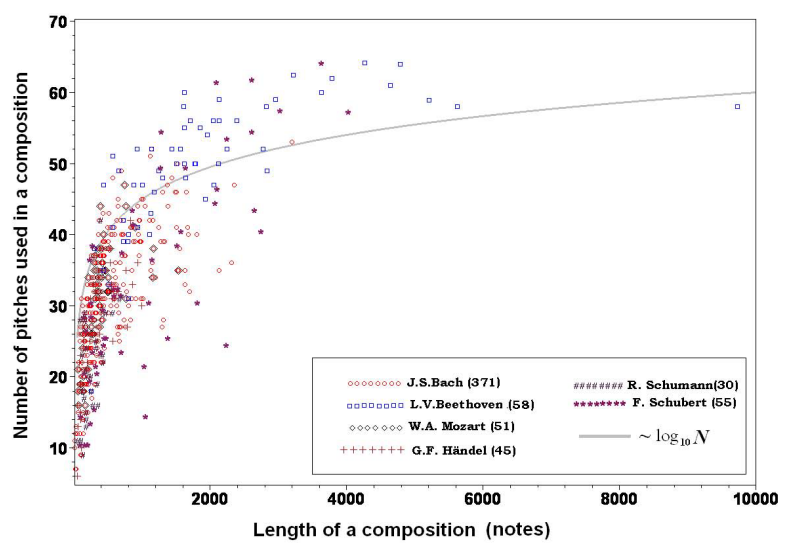

Fig. 1. The number of different pitches used in a composition grows approximately logarithmically with its size. The data have been collected over 371 compositions of J.S. Bach, 58 various compositions of L.V. Beethoven, 55 compositions of F. Schubert, 51 compositions of W.A. Mozart, 45 compositions of G.F. Händel, and 30 compositions of R. Schumann.

logarithmic curve indicating that $R \simeq \log N$ can be used as the simplest parameter assessing complexity of a musical composition.

In the MDG, we consider a note as an elementary event providing a natural discretization of musical phenomena that facilitate their performance and analysis. Namely, given the entire keyboard $\mathcal{K}$ of 128 notes (standard for the MIDI representations of music) corresponding to a pitch range of 10.5 octaves, each divided into 12 semitones, we regard a note as a discrete random variable $X$ that maps the musical event to a value of a $R$-set of pitches $\mathcal{P}=\left\{x_{1}, \ldots, x_{R}\right\} \subseteq \mathcal{K}$. In the musical dice game, a piece is generated by patching notes $X_{t}$ taking values from the set of pitches $\mathcal{P}$ that sound good together into a temporal sequence $\left\{X_{t}\right\}_{t \geq 1}$. Herewith, two consecutive notes, in which the second pitch is a harmonic of the first one are considered to be pleasing to the ear, and therefore can be patched to the sequence. Harmony is based on consonance, a concept whose definition changes permanently in musical history. Two or more notes may sound consonant for various reasons such as luck of perceptual roughness, spectral similarity of the sequence to a harmonic series, familiarity of the sound combination in contemporary musical contexts, and eventually for a personal taste, as there are consonant and dissonant harmonies, both of which are pleasing to the ears of some and not others. A detailed statistical analysis of subtle harmony conveyed by melodic lines in tonal music certainly calls for the complicated stochastic models, in which successive notes in the sequence $\left\{X_{t}\right\}_{t \geq 1}$ are not chosen independently, but their probabilities depend on preceding notes. In the general case, a set of $n$-note probabilities $\operatorname{Pr}\left[X_{t+1}=x \mid X_{t}=y, X_{t-1}=z, \ldots, X_{t-n}=v\right]$ might be required to insure the resemblance of the musical dice games to the original compositions. However, it is rather difficult to decide a priori upon the enough memory depth $n$ in 
Table 1. MIDI events for the note C4.

\begin{tabular}{l|l|l|l|l}
\hline event type & time & channel & note & velocity \\
\hline note_on & 192 & 0 & 60 & 127 \\
note_off & 192 & 0 & 60 & 64
\end{tabular}

the stochastic models required to compare reliably the pieces of tonal and atonal music created by different composers, with various purposes, in different epochs, for diverse musical instruments subjected to the dissimilar tuning techniques. Under such circumstances, it is mandatory to identify some meaningful blocks of musical information and to detect the hierarchical tonality (basic for perception of harmony in Western music ${ }^{15}$ ) in a simplified statistical model, as the first step of statistical analysis. For this purpose, in the present work, we neglect possible statistical influences extending over than the only preceding note and limit our analysis to the simplest time - homogeneous model called Markov chain ${ }^{16}$,

$$
\operatorname{Pr}\left[X_{t+1}=x \mid X_{t}=y, X_{t-1}=z, \ldots\right]=\operatorname{Pr}\left[X_{t+1}=x \mid X_{t}=y\right]=T_{y x},
$$

where the elements of the stochastic transition matrix $T_{y x}, \sum_{x \in \mathcal{P}} T_{y x}=1$, weights the chance of a pitch $x$ going directly to another pitch $y$ independently of time.

It is worth mentioning that the model (1) obviously does not impose a severe limitation on melodic variability, since there are many possible combinations of notes considered consonant, as sharing some harmonics and making a pleasant sound together. The relations between notes in (1) are rather described in terms of probabilities and expected numbers of random steps than by physical time. Thus the actual length $N$ of a composition is formally put $N \rightarrow \infty$, or as long as you keep rolling the dice. Markov chains are widely used in algorithmic music composition, as being a standard tool, in music mix and production software ${ }^{17,18,19}$.

\section{Encoding of a discrete model of music (MIDI) into a transition matrix}

While analyzing the statistical structure of musical pieces, we used the MIDI representations providing a computer readable discrete time model of music by a sequence of the 'note' events, note_on and note_off:

In the MIDI representation, each note event (like that one shown in Tab. 1) is characterized by the four variables: 'time', 'channel', 'note', and 'velocity'. Motivated by the logarithmic pitch perception in humans, music theorists represent pitches using a numerical scale based on the logarithm of fundamental frequency,

$$
\text { note }=69+12 \times \log _{2}\left(\frac{f}{440 \mathrm{~Hz}}\right) .
$$

The resulting linear pitch space in which octaves have size 12, semitones have size 1, and the number 69 is assigned to the note A4. The linear distance in the pitch space corresponds to the musical distance as measured in psychological experiments and allows a MIDI file to have a specific value of discreteness 'ticks/quarter' indicating 
Table 2. MIDI note ID numbers corresponding to musical notation.

\begin{tabular}{l|l|l|l|l|l|l|l|l|l|l|l|l}
\hline Octave & C & C\# & D & D\# & E & F & F\# & G & G\# & A & A\# & B \\
\hline 3 & 48 & 49 & 50 & 51 & 52 & 53 & 54 & 55 & 56 & 57 & 58 & 59 \\
4 & 60 & 61 & 62 & 63 & 64 & 65 & 66 & 67 & 68 & 69 & 70 & 71 \\
5 & 72 & 73 & 74 & 75 & 76 & 77 & 78 & 79 & 80 & 81 & 82 & 83
\end{tabular}

the number of 'ticks' that make up a quarter note. The value of 'time' then gives the number of 'ticks' between two consequent note events. In the example given in Tab. 1, the event of C4 starts after 192 'ticks' have passed. The 'channel' indicate one of 16 channels ( 0 to 15 ) this event may belong to.

Notes are not encoded by their names like $\mathrm{C}$ or $\mathrm{A}$. Instead, the harmonic scale is mapped onto numbers from 0 to 127 with chromatic steps. For instance, the identification number 60 corresponds to the $\mathrm{C} 4$, in musical notation. Then, note number 61 is C4\#, 62 is D4 etc. (see Tab. 2 for some octaves and their MIDI note ID numbers)

Finally, the 'velocity' $(0-127)$ describes the strength with which the note is played. As MIDI files contain all musically relevant data, it is possible to determine the probabilities of getting from one note to another for all notes in a musical composition by analyzing its MIDI file with a computer program. To get transition matrices (1) for tonal sequences, we need only 'time', 'channel', and 'note' to be considered.

The MIDI files of 804 musical compositions were processed by a program written in Perl; the MIDI parsing was done using the module Perl : :MIDI ${ }^{20}$, which allowed the conversion of the MIDI data into a more convenient form called MIDI: : Score where each two consequent note_on and note_off events are combined to a single note event. Each note event contains an absolute time, the starting time of the event, and a duration which gives the duration of the event in ticks.

To give an example of the process of getting to a transition matrix from a musical score, we consider the first bars of the fugue from BWV846 of J.S. Bach shown in Fig. 2. The numbers below the first notes in Fig. 2 indicate the corresponding MIDI ID note numbers. In Tab. 3, we show the representation of the first three notes in MIDI and in MIDI: : Score format. Here, the value of velocity is omitted.

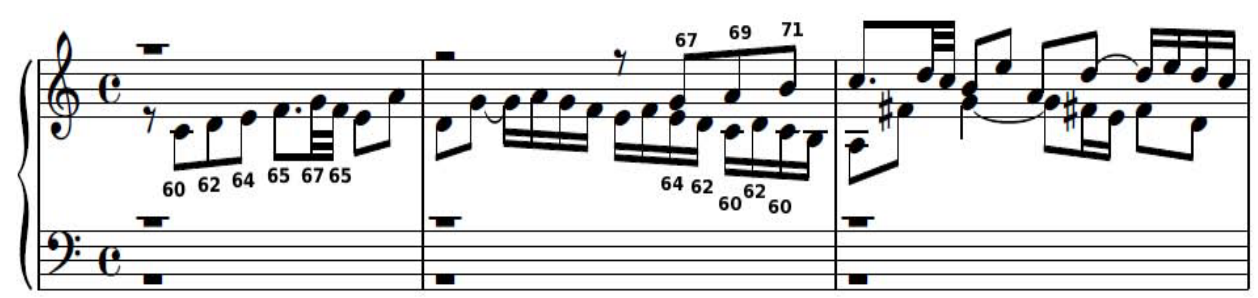

Fig. 2. The first three bars from the fugue of BWV846. Also shown are MIDI note numbers . 
Table 3. MIDI and MIDI::Score data from the beginning of fugue I BWV846.

\begin{tabular}{l|l|l|l||l|l|l|l|l}
\hline \multicolumn{2}{l|}{ "MIDI" } & \multicolumn{2}{|c}{ "MIDI::Score format" } \\
\hline event type & time & ch & note & event type & time & dur & ch & note \\
\hline note_on & 192 & 0 & 60 & note & 192 & 192 & 0 & 60 \\
note_off & 192 & 0 & 60 & & & & & \\
note_on & 0 & 0 & 62 & note & 384 & 192 & 0 & 62 \\
note_off & 192 & 0 & 62 & & & & & \\
note_on & 0 & 0 & 64 & note & 576 & 192 & 0 & 64 \\
note_off & 192 & 0 & 64 & & & & & \\
\hline
\end{tabular}

Table 4. MIDI::Score data from the middle of the second bar of fugue I BWV846 where the second voice starts playing. The note names and the voices of the events are also shown.

\begin{tabular}{clll||ll}
\hline time & dur & ch & note & name & voice \\
\hline \hline 2496 & 192 & 0 & 67 & G4 & upper \\
2496 & 96 & 0 & 64 & E4 & lower \\
2592 & 96 & 0 & 62 & D4 & lower \\
2688 & 192 & 0 & 69 & A4 & upper \\
2688 & 96 & 0 & 60 & C4 & lower \\
2784 & 96 & 0 & 62 & D4 & lower \\
2880 & 192 & 0 & 71 & B4 & upper \\
2880 & 96 & 0 & 60 & C4 & lower
\end{tabular}

For the first notes shown in Fig. 2, the definition of a transition is easy as there is only one voice. In particular, from Tab. 3, we can conclude that there would be the consequent transitions $60 \rightarrow 62$ and $62 \rightarrow 64$. However, like most musical pieces, this Fugue then contains several voices that play simultaneously, so that an additional convention is required to define a transition from note to note.

In the middle of the second bar shown in Fig. 2, a second voice is starting. Some note events starting from there are given in Tab. 4 in MIDI : Score form. From Tab. 4, it is clear that for a MIDI representation it is not necessary to put the upper voice into a different channel than that of the lower voice. In the example shown in Tab. 4, the notes 67 and 64 both start at time 2496. As note 64 has a duration of 96 ticks, it is obvious that note 62 at time 2592 belongs to the same voice as note 64 . However, for the notes 69 and 60 starting at 2688, it is unclear to which voice each note belongs to, and how they might be encoded into a transition matrix. It is important to note that such an ambiguity is not a problem of MIDI representation itself, but rather of music. It depends upon the experience of a listener how she distinguishes voices while listening to a musical composition that contains several simultaneous voices. Even if the musical score explicitly separates those voices by placing them atop of each other, our personal impression of them might not coincide with that one notated, rather arising from live audio mixing of all simultaneous voices during the performance. Thus, to get transition matrices from 
MIDI fies, we have to answer the following important question: "Which transitions between which note events have to be accounted?"

In our approach, we sort note events ascending by time and channel. By surfing over the list of events, a transition between two subsequent occurrences is accounted when the moment of time of the second event is greater than that of the first one. When several events occur simultaneously, we give a priority to the event belonging to a small number channel. Let us emphasize that under the used method not all possible transitions between note events contribute into the transition matrix.

For example, let us consider the notes shown in Fig. 3; their list of events is given in the adjacent table. The resulting transitions accounted in the matrix would be those between events marked with ${ }^{*}{ }^{*}: 76 \rightarrow 59,59 \rightarrow 60,60 \rightarrow 78,78 \rightarrow 72$. Note events with small channel values are favored over those with higher values. For simultaneous note events occurring in the same channel, only the first one is considered that mostly means the topmost voice, in musical notation.

We believe that the encoding method we use is quite efficient for unveiling the individual melodic lines and identifying a creative character of a composer from musical compositions because of the appearance of the resulting transition matrices. Those matrices generated with respect to the chosen encoding method look differently, from piece to piece and from composer to composer (see the examples shown in Fig. 4). However, if we were treated each voice in a musical composition separately (the transitions of the upper voice and those of the lower voice might be accounted independently while computing the probabilistic vector forming a row of the transition matrix), the transition matrices were clearly dominated by a region along the main diagonal, similarly for all compositions. The last but not least remark upon the second algorithm is that although the statistics of transitions in those matrices is much more rich, they formally account for a number of odd transitions between the simultaneous voices definitely inconsistent with the main melody. It is important to mention that no matter which encoding method is used the resulting transition matrices appear to be essentially not symmetric: if

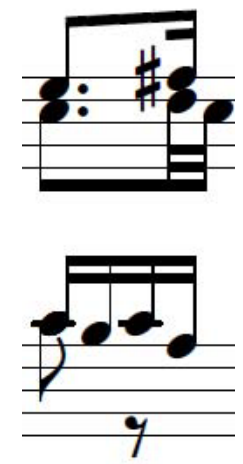

\begin{tabular}{llll|ll} 
time & dur & ch & note & name & \\
\hline \hline 13056 & 288 & 0 & 76 & (E5) & $*$ \\
13056 & 288 & 0 & 72 & (C5) & \\
13056 & 192 & 1 & 60 & (C4) & \\
\hline 13152 & 96 & 1 & 59 & (B3) & $*$ \\
13248 & 96 & 1 & 60 & $(\mathrm{C} 4)$ & $*$ \\
\hline 13344 & 96 & 0 & 78 & $(\mathrm{~F} \# 5)$ & $*$ \\
13344 & 48 & 0 & 74 & $(\mathrm{D} 5)$ & \\
13344 & 96 & 1 & 57 & (A3) & \\
\hline 13392 & 48 & 0 & 72 & (C5) & $*$
\end{tabular}

Fig. 3. Example from our fugue ${ }^{13}$. 
F. Chopin, Revolutionary Etude. Op.10 No.12

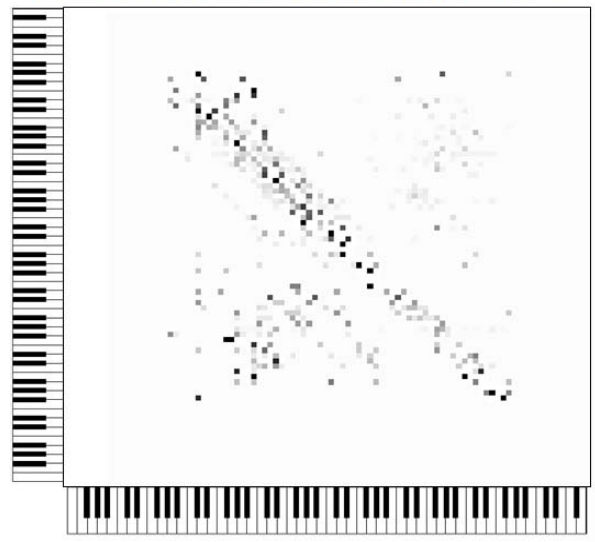

I. Stravinsky, The Firebird

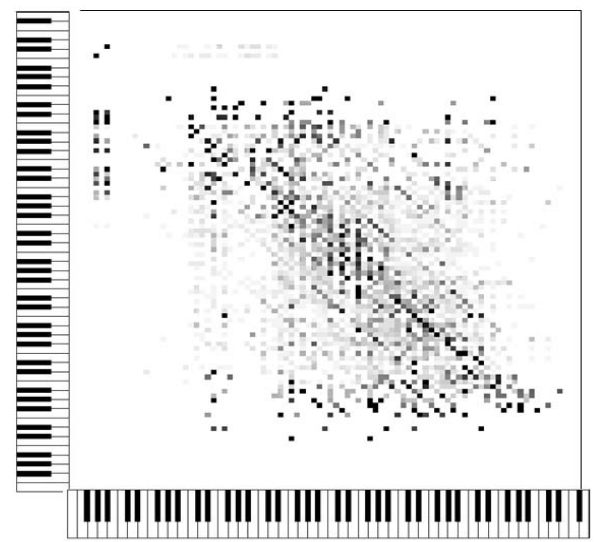

Fig. 4. Transition matrices for the MDG based on the F.Chopin "Revolutionary Etude" (Op.10, No 12) (left) and the I. Stravinsky "The Fire-bird" suite (right).

$T_{x y}>0$, for some $x, y$, it might be that $T_{y x}=0$. A musical composition can be represented by a weighted directed graph, in which vertices are associated with pitches and directed edges connecting them are weighted accordingly to the probabilities of the immediate transitions between those pitches. Markov's chains determining random walks on such graphs are not ergodic: it may be impossible to go from every note to every other note following the score of the musical piece.

\section{Musical dice game as a generalized communication process}

A musical piece generated as an output of the musical dice game (1) is a stochastic process which can be encoded by a sequence of independent and identicallydistributed random variables representing notes which can take values of different pitches. To measure the uncertainty associated with a pitch in such a stochastic process, we can use the Shannon entropy ${ }^{21}$,

$$
H=-\sum_{x \in \mathcal{P}} \pi_{x} \log _{R} \pi_{x}
$$

where $\pi_{x}$ is the probability to find the note $x \in \mathcal{P}$ in the musical score, and the base of the logarithm is $R=|\mathcal{P}|$. Since the entropy of a musical piece defined by (3) is affected by the number of used pitches $R$, the parameter of information redundancy, $\mathcal{R}=1-H / \max H, \quad \max H=\log R$, where $\max H$ is the theoretical maximum entropy, might be used for comparing different musical compositions. Accordingly to information theory ${ }^{22}$, redundancy quantifies predictability of a pitch in the piece, as being a natural counterpart of entropy.

As we have mentioned above, a Markov chain encoding the musical dice game might be not ergodic, and therefore the probability to find a pitch in the musical 
score cannot be found simply as the entry in the left eigenvector (which now might be non-unique) of the transition matrix $\mathbf{T}$ belonging to the maximal eigenvalue $\mu=1$. In order to find the probability of observing the note in the musical score, we can use the method of group generalized inverse ${ }^{23,24}$ that might be applied for analyzing every Markov chain regardless of its structure. As the Laplace operator corresponding to the Markov chain (1), $\mathbf{L}=\mathbf{1}-\mathbf{T}$, where $\mathbf{1}$ is a unit matrix, is always a member of a multiplicative matrix group, it always possesses a group inverse $\mathbf{L}^{\sharp}$, a special case of the Drazin generalized inverse $e^{23,25,26}$ satisfying the Erdélyi conditions ${ }^{27}$ :

$$
\mathbf{L L}^{\sharp} \mathbf{L}=\mathbf{L}, \quad \mathbf{L}^{\sharp} \mathbf{L} \mathbf{L}^{\sharp}=\mathbf{L}^{\sharp}, \quad\left[\mathbf{L}, \mathbf{L}^{\sharp}\right]=0,
$$

where $[\mathbf{A}, \mathbf{B}]=\mathbf{A B}-\mathbf{B A}$ denotes the commutator of the two matrices. The methods for computing the group generalized inverse for matrices of $\operatorname{rank}(\mathbf{L})=N-1$ by considering the eigenprojection of the matrix $\mathbf{L}$ corresponding to the smallest eigenvalue $\lambda_{1}=0$ have been developed in ${ }^{28,29,30}$,

$$
\mathbf{L}^{\sharp}=(\mathbf{L}+\mathbf{Z})^{-1}-\mathbf{Z}, \quad \mathbf{Z}=\prod_{\lambda_{i} \neq 0}\left(\mathbf{1}-\frac{1}{\lambda_{i}} \mathbf{L}\right)
$$

where the product in the idempotent matrix $\mathbf{Z}$ is taken over all nonzero eigenvalues of $\mathbf{L}$. The role of group inverses (4) in the analysis of Markov chains has been discussed in details in ${ }^{23,24,31}$. Here, we only mention that a generalized inverse provides the unique best fit (with respect to least squares) approximation for a solution (among infinitely many) to the system of linear equations described by the matrix $\mathbf{L}^{-1}$. Then the correspondent best fit approximation to the stationary distribution can be calculated as

$$
\pi_{x_{i}}=\left(\mathbf{1}-\mathbf{L} \mathbf{L}^{\sharp}\right)_{x_{i} x_{j}} ;
$$

the rows of (6) are all equal to the corresponding components of the vector $\pi$. It is important to mention that in the framework of the method of group generalized inverses, we account for all possible sequences of pitches that would arise if we start the musical dice game infinitely many times from the randomly chosen notes. Thus, the inverse value $\pi_{x_{i}}^{-1}$ calculated in accordance to (6), i.e. the recurrence time to the state $x_{i}$, would formally be finite even if the state is transient (i.e., for which there is a non-zero probability that we will never return to the state); in the same way, the recurrence time would not equal zero for absorbing states (which are impossible to leave).

Determining the entropy of texts written in a natural language is an important problem of language processing. The entropy of current written and spoken languages (English, Spanish) has been estimated experimentally as ranged from 0.5 to 1.3 bit per character ${ }^{2,32}$. An approximately even balance (50:50) of entropy and redundancy is supposed as necessary to achieve effective communication in transmitting a message, as it makes easier for humans to perceive information ${ }^{32}$. 

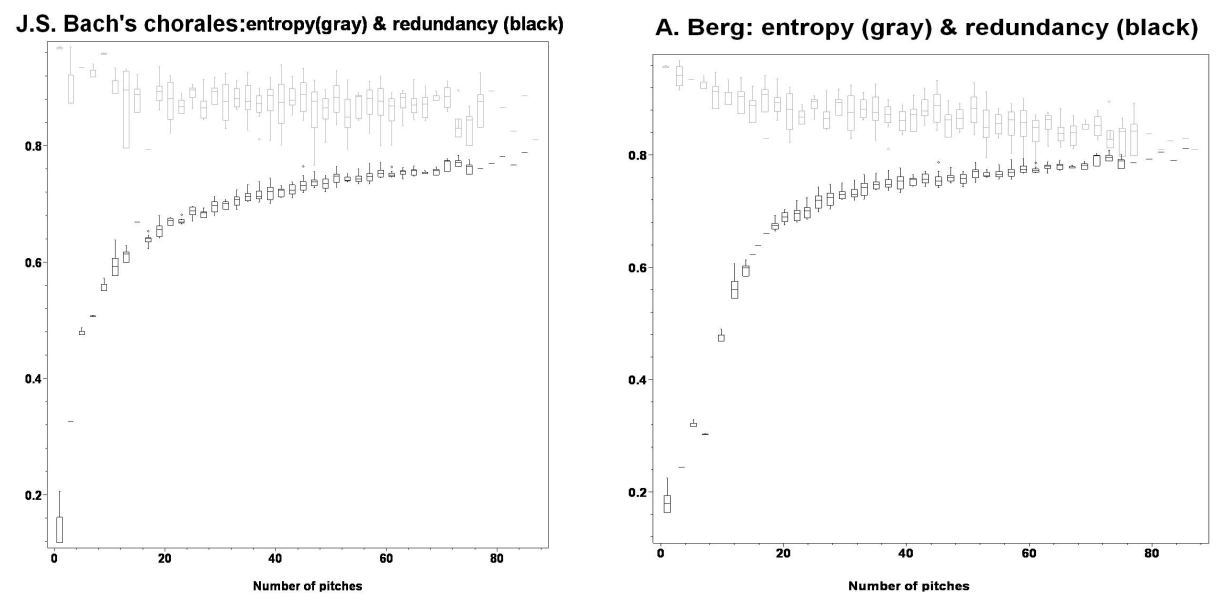

Fig. 5. The box plots show the statistic of the magnitudes of entropy and redundancy vs. the number of pitches used in a composition, for the MDG generated over the 371 compositions of J.S. Bach (left) and the 7 compositions of A. Berg (right). In a box plot, a central line of each box shows the median; a lower line shows the first quartile; an upper line shows the third quartile; two lines extending from the central box of maximal length $3 / 2$ the interquartile range but not extending past the range of the data; eventually, the outliers are those points lying outside the extent of the previous elements.

For all MDG we studied, the magnitudes of entropy fluctuate in a range between 0.7 and 1.1 bit per note well fitting with the entropy range of usual languages. It is important to note that pieces involving more pitches appear to be characterized with lower magnitudes of entropy but higher values of redundancy (predictability). In Fig. 5, we have presented the statistics of entropy and redundancy vs. the number of pitches through their five-number summaries, for 371 chorales of J.S. Bach (left) and 7 compositions of A. Berg (right). A central line of each box in the box plot (Fig. 5) shows the median (not the mean), the value separating the higher half of the data sample from the lower half, that is found by arranging all the observations from lowest value to highest value and picking the middle one. Other lines of the box plot indicate the quartile values which divide the sorted data set into four equal parts, so that each part represents one fourth of the sample. A lower line in each box shows the first quartile, and an upper line shows the third quartile. Two lines extending from the central box of maximal length $3 / 2$ the interquartile range but not extending past the range of the data. The outliers are those points lying outside the extent of the previous elements.

The entropy and redundancy statistics shown in Fig. 5 suggests that musical compositions generated as an output of the MDG might contain some repeated patterns, or motives in which certain combinations of notes are more likely to occur than others. In particular, the dramatic increase of redundancy as the range of pitches expands up to 7.5 octaves implies that musical compositions involving 
many pitches might convey mostly conventional, predictable blocks of information to a listener. However, in contrast to human languages where entropy and redundancy are approximately equally balanced ${ }^{32}$, in the MDG pieces based on classical music entropy clearly dominates over redundancy. While decoding a musical message requires the listener to invest nearly as much efforts as in everyday decoding of speech, the successful understanding of the composition would call for an experienced listener ready to invest his or her full attention to a communication process that would span across cultures and epochs.

Another possible information measure that can be applied to the analysis of MDG is the past-future mutual information (complexity) introduced in studies of the symbolic sequences generated by dynamical systems ${ }^{33}\left(\right.$ see also $\left.^{22}\right)$. It estimates the information content of the patches of notes and can be formally derived as the limiting excess of the block entropy

$$
H\left(S^{m}\right)=-\sum_{S^{m}} P\left(S^{m}\right) \log _{R} P\left(S^{m}\right),
$$

in which $P\left(S^{m}\right)$ is the probability to find a patch $S^{m}$ of $m$ notes, over the $m$ times Shannon entropy $H$, as the size of the block $m \rightarrow \infty$,

$$
C=\lim _{m \rightarrow \infty}\left(H\left(S^{m}\right)-m H\right) .
$$

Following ${ }^{34}$, we use the fact that the transition probability between states in a Markov chain determined by the matrix (1) is independent of $m$, so that complexity (7) can be computed simply as

$$
C=-\sum_{x \in \mathcal{P}} \pi_{x} \log _{R} \frac{\pi_{x}}{\prod_{y \in \mathcal{P}} T_{x y}^{T_{x y}}} .
$$

In Fig. 6 (left), we have presented the statistics of complexity values for the random pieces generated by the MDG based on Bach's compositions. The main trend (shown in Fig. 6 (left) by a solid line) represents the cubic splines interpolating between the mean (not the median) complexity values and indicates that patches consisting of 8 notes are characterized by the maximum past-future mutual information. Then complexity decreases rapidly with the number of pitches used in a composition suggesting that the musical pieces might contain a few types of melodic prototypes translated over the entire diapason of pitches by chromatic transposition. Finally, in Fig. 6 (right), we have sketched a scatter plot showing the pace of complexity with entropy in the MDG generated over the 480 compositions of classical music that implies that a strong positive correlation exists between the value of entropy and the logarithm of complexity, in those compositions.

\section{First passage times to notes resolve tonality of musical dice games}

Statistics of entropy, redundancy, and complexity in the MDG over classical musical compositions suggests that tonal music generated by the musical dice game (1) constitutes the well structured data that contain conventional patterns of information. 

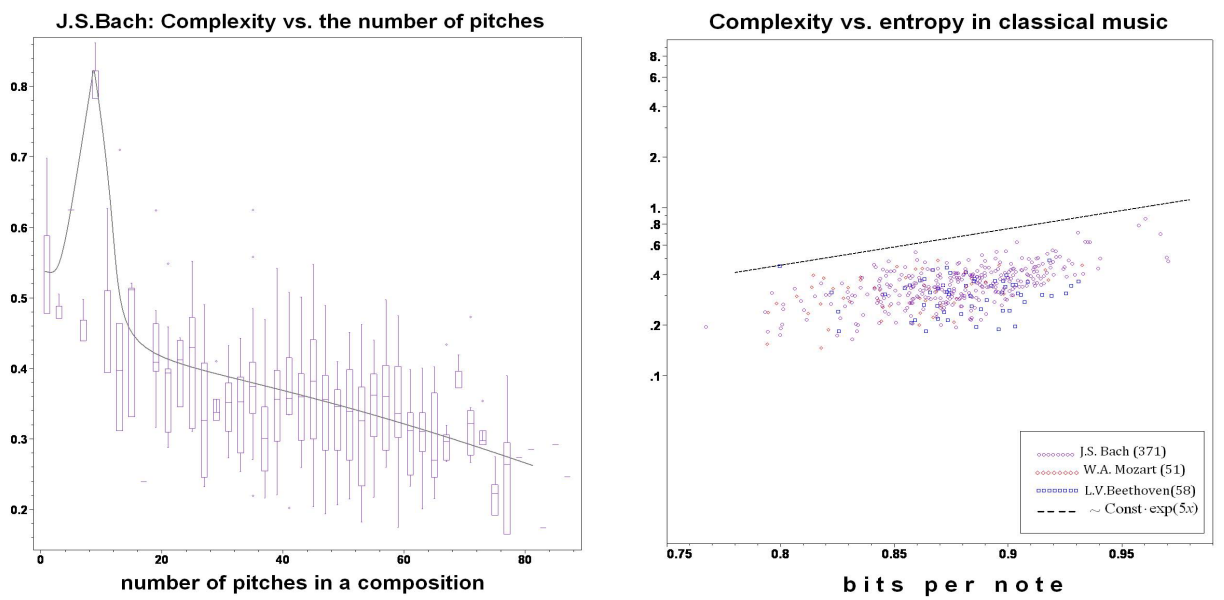

Fig. 6. The box plot (left) represents complexity (measured by the past-future mutual information) vs. the number of pitches used in a composition, for the MDG inspired by the 371 pieces of J.S. Bach. The trend (shown by a solid line) stands for the cubic splines interpolating between the mean values of complexity over the data ranges. The scatter plot of complexity vs. the magnitude of entropy in 480 pieces generated by the MDG (given in the log-linear scale) suggests that a strong positive correlation exists between the value of entropy and the logarithm of complexity. The reference line indicates an exponential growth, in the log-linear scale.

Obviously, some notes might be more "important" than others, with respect to such a structure.

In music theory ${ }^{35}$, the hierarchical pitch relationships are introduced based on a tonic key, a pitch which is the lowest degree of a scale and that all other notes in a musical composition gravitate toward. A successful tonal piece of music gives a listener a feeling that a particular (tonic) chord is the most stable and final. The regular method to establish a tonic through a cadence, a succession of several chords which ends a musical section giving a feeling of closure, may be difficult to apply without listening to the piece.

While in a MDG, the intuitive vision of musicians describing the tonic triad as the "center of gravity" to which other chords are to lead acquires a quantitative expression. Namely, every pitch in a musical piece is characterized with respect to the entire structure of the Markov chain by its level of accessibility estimated by the first passage time to it ${ }^{36,37}$ that is the expected length of the shortest path of a random walk toward the pitch from any other pitch randomly chosen over the musical score. Analyzing the first passage times in scores of tonal musical compositions, we have found that they can help in resolving tonality of a piece, as they precisely render the hierarchical relationships between pitches. It is interesting to note that from the physical point of view the first passage time can be naturally interpreted as a potential, as being equal to the diagonal elements of the generalized inverse of the Laplace operator. For example, the first passage time to a node precisely equals 
to the electric potential of the node, in an electric resistance network ${ }^{37,38}$. Thus, in the framework of musical dice games, the role of a note in a tonal scale can be understood as its potential.

The majority of tonal music assumes that notes spaced over several octaves are perceived the same way as if they were played in one octave ${ }^{39}$.

Using this assumption of octave equivalency, we can chromatically transpose each musical piece into a single octave getting the $12 \times 12$ transition matrices, uniformly for all musical pieces, independently of the actual number of pitches used in the composition. Given a stochastic matrix $\mathbf{T}$ describing transitions between notes within a single octave $\mathcal{O}$, the first passage time to the note $i \in \mathcal{O}$ is computed ${ }^{37}$ as the ratio of the diagonal elements,

$$
\mathcal{F}_{i}=\left(\mathbf{L}^{\#}\right)_{i i} /\left(\mathbf{1}-\mathbf{L} \mathbf{L}^{\#}\right)_{i i},
$$

where $\mathbf{L}$ is the Laplace operator corresponding to the transition matrix $\mathbf{T}$, and $\mathbf{L}^{\#}$ is its group generalized inverse. Let us note that in the case of ergodic Markov chains the result (9) coincides with the classical one about the first passage times of random walks defined on undirected graphs ${ }^{40}$.

In Fig. 7, we have shown the two examples of the arrangements of first passage times to notes in one octave, for the E minor scale (left) and E major, A major scales (right). The basic pitches for the E minor scale are E, F\#, G, A, B, C, and D. The E major scale is based on E, F\#, G\#, A, B, C\#, and D\#. Finally, the A major scale consists of A, B, C\#, D, E, F\#, and G\#. The values of first passage times are strictly
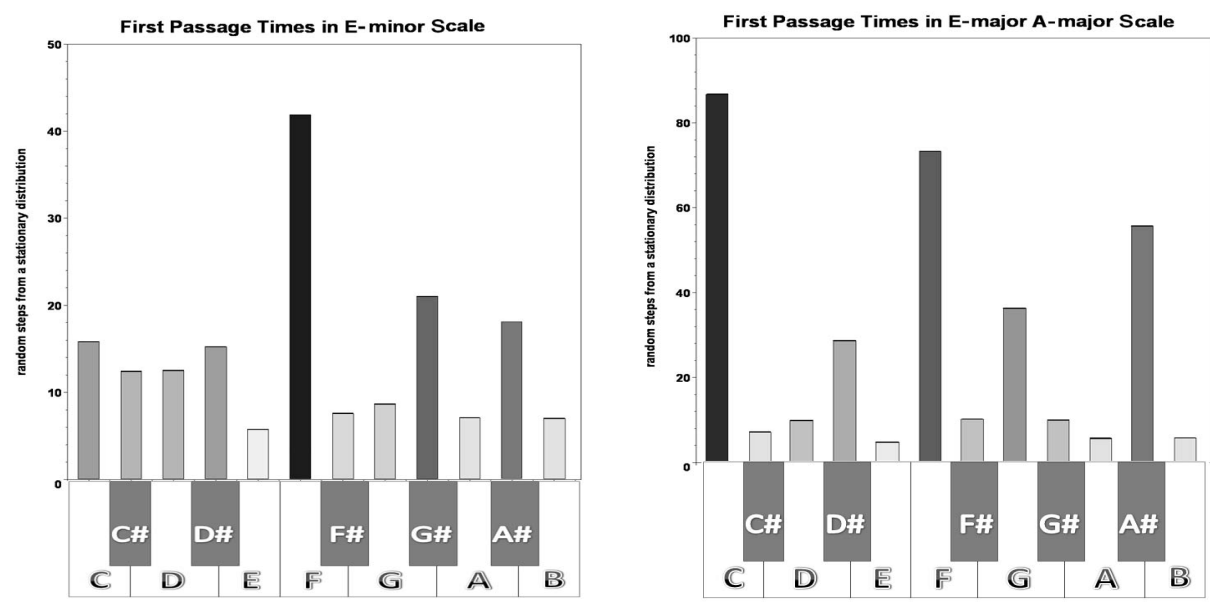

Fig. 7. The histograms show the first passage times to the notes for the MDG over a part of Duet I of J.S. Bach (BWV 802) written in E minor (left) and over a part of the Cello Sonata No.3, Op.69 of L.V. Beethoven written in E major, A major (right) mapped into a single octave. Bars are shaded with the intensity of gray scale $0-100 \%$, in proportion to the magnitude of the first passage time. Therefore, the basic pitches of a tonal scale are rendered with light gray color, as being characterized by short first passage times, and the tonic key by the smallest magnitude of all. 
ordered in accordance to their role in the tone scale of the musical composition. Herewith, the tonic key is characterized by the shortest first passage time (usually ranged from 5 to 7 random steps), and the values of first passage times to other notes collected in ascending order reveal the entire hierarchy of their relationships in the musical scale.

It is intuitive that the time of recurrence to a note estimated by $\pi_{i}^{-1}$ is related positively to the first passage time to it, $\mathcal{F}_{i}$ : the faster a random walk over the score hits the pitch for the first time, the more often it might be expected to occur again. The time of recurrence equals the first passage time in a salient recurring succession of notes (a motif) the pattern of three short notes followed by one long that opens the Fifth Symphony of L.V. Beethoven and reappears throughout the work is a classic example.

The log-log scatter plot shown in Fig. 8 represents the relation between the recurrence time and the first passage time to the 12 notes of one octave in all MDG over musical compositions we studied. The straight line indicates equality of recurrence times and first passage times. The data provide convincing evidence for the systematic departure of recurrence times from first passage times for those pitches characterized by the relatively short first passage times (recurrence times to them are typically longer than first passage times). The excess of recurrence times over first passage times quantifies musical development encompassing distinct musical figures that are subsequently altered and sequenced throughout a piece of music. It is not a surprise that such a musical development is essentially visible

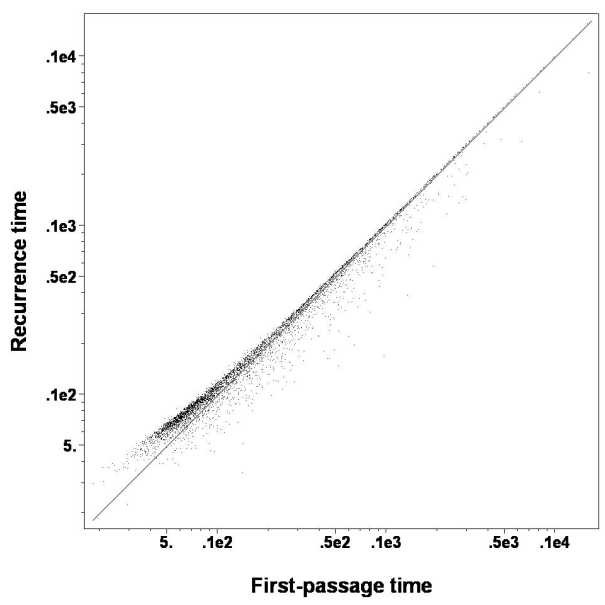

Fig. 8. The log-log scatter plot contains $12 \times 804$ points representing the recurrence time vs. the first passage time to the 12 notes of one octave, over the MDG based on 804 compositions of 29 composers. The straight line is given for a reference indicating the horizon of intelligibility (when equality of recurrence times and first passage times is achieved); departures from linearity signify departures from intelligibility. 
in the range of the relatively short first passage times, as they play an important role in the tonal scale structure of a piece guaranteeing its unity. The important conclusion of this section is that the frequency analysis of note occurrences alone is not complete enough to reliably resolve the tonality of a musical composition.

\section{First passage times to notes feature a composer}

By analyzing the typical magnitudes of first passage times to notes in one octave, we can discover an individual creative style of a composer and track out the stylistic influences between different composers.

The box plots shown in Fig. 9 depict the data on first passage times to notes in the arrangements generated by the MDG over a number of compositions written by J.S. Bach, A. Berg, F. Chopin, and C.Franck through their five-number summaries: $3 / 2$ the interquartile ranges, the lower quartile, the third quartile, and the median. In tonal music, the magnitudes of first passage times to the notes are completely determined by their roles in the hierarchy of tone scales. Therefore, a low median in the box plot (Fig. 9) indicates that the note was often chosen as a tonic key in many compositions.

Correlation and covariance matrices calculated for the medians of the first passage times in a single octave provide the basis for the classification of composers, with respect to their tonality preferences. For our analysis, we have selected only those musical compositions, in which all 12 pitches of the octave were used. The tone scale symmetrical correlation matrix has been calculated for 23 composers, with the elements equal to the Pearson correlation coefficients between the medians of the first passage times. For exploratory visualization of the tone scale correlation matrix, we arranged the "similar" composers contiguously. Following ${ }^{41}$, while ordering the composers, we considered the eigenvectors (principal components) of the correlation matrix associated with its three largest eigenvalues. Since the cosines of angles between the principal components approximate the correlations between the tonal preferences, we used an ordering based on the angular positions of the three major eigenvectors to place the most similar composers contiguously, as it is shown in Fig. 10. The correlogram presented on Fig. 10 allows for identifying the three groups of composers exhibiting similar preferences in the use of tone scales, as correlations are positive and strong within each tone group while being weak or even negative between the different groups. The smaller subgroups might be seen within the first largest group (from J. Strauss to G. Fauré), in the left upper corner of the matrix on Fig. 10. Most of the composers that appeared in the largest group are traditionally attributed to the Classical Period of music. The strongest positive correlations we observed in the choice of a tonic key (about 97\%) is between the compositions of J. Strauss and A. Vivaldi who led the way to a more individualistic assertion of imaginative music. The tonality statistics in the masterpieces of R. Wagner appears also quite similar to them. Other subgroups are formed by G.F. Händel and D. Shostakovitch, J.S. Bach and R. Schumann. The Classical Period boasted 

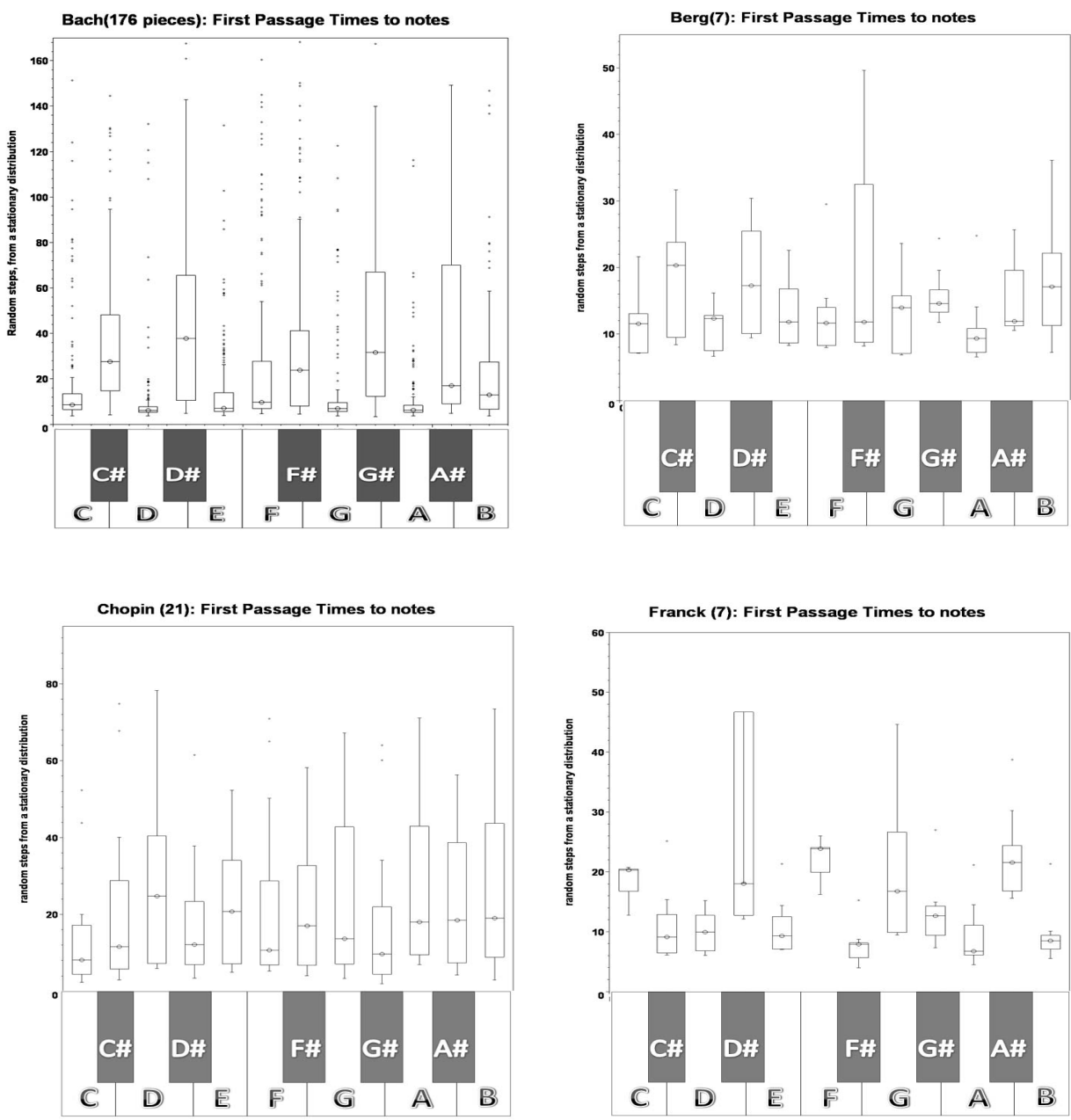

Fig. 9. Statistics of first passage times of in the musical pieces generated by the MDG over the original compositions of J.S. Bach, A. Berg, F. Chopin, and C.Franck are represented through their five-number summaries in the box plots.

by L.V. Beethoven and W.A. Mozart who led the way further to the Romantic period in classical music. F. Mendelssohn Bartholdi was deeply influenced by the music of J.S. Bach, L.V. Beethoven, and W.A. Mozart, as often reflected by his biographers ${ }^{42}$ - not surprisingly, he found his place next to them. Furthermore, the piano concerts of C. Saint-Saëns were known to be strongly influenced by those of W.A. Mozart, and, in turn, appear to have influenced those of S. Rachmaninoff that receives full exposure in the correlogram (Fig. 10). Moreover, we also get the evidence of affinity between I.Stravinsky and A. Berg, F. Schubert, F. Chopin, and G. Faure, as well as of the strong correlation between the tonality styles of A. Scriabin 


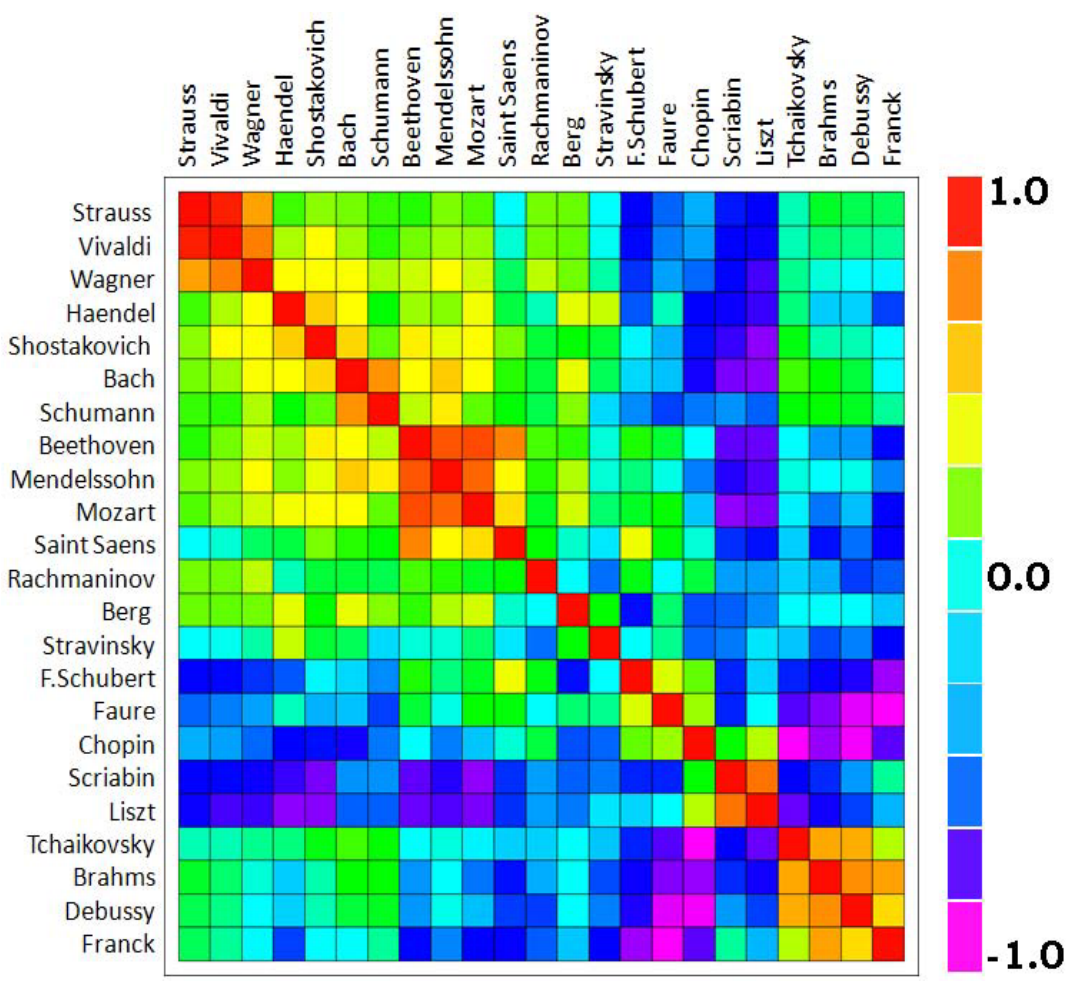

Fig. 10. The correlogram displays the correlation matrix for the medians of the first passage times to notes of one octave, for 23 composers. In the shaded rows, each cell is shaded from violet to red depending on the sign of the correlation, and with the intensity of color scaled $0-100 \%$, in proportion to the magnitude of the correlation.

and F. Liszt. The last group, in the lower right conner of the matrix are occupied by the Middle and Late Romantic era composers: P. Tchaikovsky, J. Brahms, C. Debussy, and C. Franck. Interestingly, the names of composers that are contiguous in the correlogram (Fig. 10) are often found together in musical concerts and on records performed by commercial musicians.

\section{Conclusions}

We have studied the MDG encoded by the transition matrices between pitches over the 804 musical compositions. Contrary to human languages where the alphabet is independent of a message, musical compositions might involve different sets of pitches; the number of pitches used to compose a piece grows approximately logarithmically with its size. Entropy dominates over redundancy in the MDG based on the compositions of classical music. Statistics of complexity in the note sequences 
generated by the MDG suggests that the maximum of past-future mutual information is achieved on the blocks consisting of just a few notes ( 8 notes, for the MDG generated over Bach's chorales) which might serve as a base for the melody prototypes. Pieces in classical music might contain a few melodic prototypes translated over the diapason of pitches by chromatic transposition. The hierarchical relations between pitches in tonal music might be rendered by means of first passage times to them. The frequency analysis of note occurrences is not enough to reliably resolve the tonality of a musical composition, since recurrence times to notes are typically longer than first passage times reflecting complex musical development throughout the musical piece. Correlations between the medians of the first passage times to the notes provide the basis for a phylogenetic classification of composers, with respect to their tonality preferences.

\section{Acknowledgments}

We thank Ph. Blanchard, T. Krüger, and J. Loviscach, for the inspiring discussions.

\section{References}

1. C.E. Shannon,Bell System Technical Journal 27, pp. 379-423; 623-656 (1948).

2. C.E. Shannon, The Bell System Technical Journal 30, p. 50 (1951).

3. J. Wolfe, "Speech and music, acoustics and coding, and what music might be 'for'." Proc. the 7th International Conference on Music Perception and Cognition, Sydney, 2002; C. Stevens, D. Burnham, G. McPherson, E. Schubert, J. Renwick (Eds.). Adelaide: Causal Productions.

4. Ch. Seeger, Ethnomusicology 15(3), p. 385 (1971).

5. L.A. Hiller, L.M. Isaacson, Experimental Music-Composition with an Electronic Computer. New York: McGraw-Hill (1959).

6. I. Xenakis, Formalized Music. Bloomington: Indiana University Press (1971).

7. K. Jones, Computer Music Journal 5 (2) (1981).

8. D. Zicarelli, Computer Music Journal 11 (4) (1987).

9. D.A. Levitt, "A Representation for Musical Dialects". In S. Schwanauer, D. Levitt (Eds.) Machine Models of Music. Cambridge, Massachusetts: The MIT Press (1993).

10. J.A. Moorer, "Music and Computer Composition". In S. Schwanauer, D. Levitt (Eds.) Machine Models of Music. Cambridge, Massachusetts: The MIT Press (1993).

11. Y. Marom, Improvising Jazz With Markov Chains The report for the Honor Program of the Department of Computer Science, The University of Western Australia (1997).

12. D.M. Franz, "Markov Chains as Tools for Jazz Improvisation Analysis". Master's Thesis, Industrial and Systems Engineering Department, Virginia Tech (1998).

13. All music in the Mutopia Project free to download, print out, perform and distribute is available at $h t t p: / / w w w . m u t o p i a p r o j e c t . o r g$. While collecting the data, we have also used

the following free resources: http://windy.vis.ne.jp/art/englib/berg.htm (for Alban Berg), http://www.classicalmidi.co.uk/page\%.htm, http://www.jacksirulnikoff.com/.

14. H. Noguchi, "Mozart: Musical game in C K.516f". Available at http://www.asahinet.or.jp/ rb5h-ngc/e/k516f.htm (1996).

15. C Dahlhaus, "Harmony". Grove Music Online, ed. L. Macy at www.grovemusic.com (2007). 
16. A.A. Markov. "Extension of the limit theorems of probability theory to a sum of variables connected in a chain". reprinted in Appendix B of: R. Howard. Dynamic Probabilistic Systems, vol. 1: Markov Chains. John Wiley and Sons (1971).

17. Native Instruments Software Synthesis GmbH, Reaktor 5.1 [computer software], Berlin.

18. Propellerhead Software, Reason 4 [computer software], Stockholm (2007).

19. A.G. Ableton, Live 8 [computer software], Berlin.

20. This software is freely available at http://search.cpan.org/šsburke/MIDI-Perl-0.8.

21. T. Schürmann, P. Grassberger, Chaos 6(3) 414-427 (1996).

22. T.M. Cover, J.A. Thomas, Elements of Information Theory. London: Wiley (1991).

23. C.D. Meyer,SIAM Rev. 17, p. 443 (1975).

24. C.D. Meyer, "Analysis of finite Markov chains by group inversion techniques. Recent Applications of Generalized Inverses", in: S.L. Campbell (Ed.), Research Notes in Mathematics 66, Pitman, Boston, p. 50 (1982).

25. M.P. Drazin, The American Mathematical Monthly 65, 506-514 (1958).

26. A. Ben-Israel, Th.N.E. Greville, Generalized inverses: theory and applications. Springer; 2nd edition (2003).

27. I. Erdélyi,J. Math. Anal. Appl. 17, 119-132 (1967).

28. S.L. Campbell, C.D. Meyer, N.J. Rose, SIAM J. Appl. Math. 31(3), 411 (1976).

29. R.E. Hartwig, SIAM J. Appl. Math. 31 (1), 42 (1976).

30. R.P. Agaev, P.Yu. Chebotarev, Automation and Remote Control 63 (10), 1537 (2002).

31. S.L. Campbell, C.D. Meyer, Generalized Inverses of Linear transformations. New York: Dover Publications (1979).

32. N. Lin, The Study of Human Communication, The Bobbs-Merrill Company, Indianapolis, (1973).

33. R. Shaw, The dripping faucet as a model chaotic system, CA Aerial Press, Santa Cruz (1984).

34. W. Li, Complex systems 5, 381 (1991).

35. W. Thomson, Tonality in Music: A General Theory., San Marino, Calif.: Everett Books (1999).

36. $\mathrm{Ph}$

Blanchard, D. Volchenkov, Proc. R. Soc. A 464, 21532167; doi:10.1098/rspa.2007.0329 (2008).

37. D. Voclchenkov, "Random walks and flights over connected graphs and complex networks", Commun. Nonlinear Sci. Numer. Simulat., doi:10.1016/j.cnsns.2010.02.016 (2010).

38. P.G. Doyle, J.L. Snell, Random Walks and Electrical Networks. Math. Assn. of America (1984); freely redistributable under the terms of the GNU General Public License (2000).

39. E.M. Burns, Intervals, Scales, and Tuning. The Psychology of Music. Second edition, Deutsch, Diana, ed. San Diego: Academic Press (1999).

40. Lovász, L. 1993 Random Walks On Graphs: A Survey. Bolyai Society Mathematical Studies 2: Combinatorics, Paul Erdös is Eighty, Keszthely (Hungary), p. 1-46.

41. M. Friendly, The American Statistician 56(4), 316 (2002).

42. C. Brown, A Portrait of Mendelssohn, New Haven and London (2003). 